Artículo original

(Original paper)

\title{
MODELAJE DE LA DISTRIBUCIÓN GEOGRÁFICA DE CUATRO ESPECIES DE SERPIENTES VENENOSAS Y SU PERCEPCIÓN SOCIAL EN EL SURESTE DE LA ALTIPLANICIE DE CHIAPAS
}

\section{MODELLING THE GEOGRAPHIC DISTRIBUTION OF FOUR VENOMOUS SNAKES AND THEIR SOCIAL PERCEPTION IN THE SOUTHEASTERN ALTIPLANICIE DE CHIAPAS}

\author{
JoRge ARTURo HIDALGO-GARCÍA ${ }^{1}{ }^{*}$, JoSÉ ROGELIo CEDEÑO-VÁZQUEZ ${ }^{1}$, RoBERTo LUNA-REYES ${ }^{2}$ \\ DAVID GONZÁLEZ-SOLÍS ${ }^{1}$ \\ ${ }^{1}$ El Colegio de la Frontera Sur (ECOSUR), Unidad Chetumal. Departamento de Sistemática y Ecología Acuática. Av. Centenario \\ km 5.5, C.P. 77014. Chetumal, Quintana Roo, México.<jahg03@hotmail.com>; <rcedenov@ecosur.mx>; \\ $<$ dgonzale@ecosur.mx> \\ ${ }^{2}$ Coordinación Técnica de Investigación, Secretaría de Medio Ambiente e Historia Natural, Calzada de los Hombres Ilustres s/n, \\ Fraccionamiento Francisco I. Madero, Col. Centro, C.P. 29000, Tuxtla Gutiérrez, Chiapas, México. <rlr07@hotmail.com> \\ *Autor de correspondencia: <jahg03@hotmail.com>
}

Recibido: 28/06/2017: aceptado: 17/03/2018; publicado en línea: 24/10/2018

Editor responsable: Gustavo Aguirre

Hidalgo-García, J. A., Cedeño-Vázquez, J. R., Luna-Reyes, R., González-Solís, D. (2018) Modelaje de la distribución geográfica de cuatro especies de serpientes venenosas y su percepción social en el sureste de la Altiplanicie de Chiapas. Acta Zoológica Mexicana (nueva serie), 34, 1-20. DOI: https://doi.org/10.21829/azm.2018.3412111

RESUMEN. Se describe la distribución geográfica de cuatro especies de serpientes venenosas (Bothriechis aurifer, Crotalus simus, Micrurus diastema y M. elegans) que habitan en el área sureste de la Altiplanicie de Chiapas. Estas especies se encuentran en alguna categoría de riesgo en la NOM-059SEMARNAT-2010, se desconoce su distribución precisa y son de importancia médica. Los modelos de distribución se obtuvieron usando el algoritmo MaxEnt, para ello se usaron registros de museos nacionales y del extranjero, así como búsquedas en internet, en la literatura científica y muestreos en campo. También se realizaron entrevistas para determinar la percepción y la relación que tienen las serpientes venenosas con las poblaciones humanas. Además, se determinó la representatividad de cada una de las especies en las áreas naturales protegidas. Algunas barreras geográficas, como el Valle de Motagua, limitan la distribución de M. elegans y B. aurifer, mientras que el Istmo de Tehuantepec lo hace para C. simus. Se pudo determinar que $B$. aurifer es la especie más vulnerable, debido a su distribución restringida, y a que se encuentra poco representada en las áreas naturales protegidas. Son frecuentes las mordeduras de serpientes venenosas en algunas localidades. La víbora de cascabel (C. simus) es perseguida por el uso medicinal que le dan los pobladores. La información generada es fundamental para implementar programas de educación ambiental y acciones de manejo que contribuyan a la conservación de estas especies.

Palabras clave: Categorías de riesgo; importancia médica; MaxEnt, entrevistas; Áreas Naturales Protegidas 
Hidalgo-García, J. A., Cedeño-Vázquez, J. R., Luna-Reyes, R. \& González-Solís, D. (2018) Modelling the geographic distribution of four venomous snakes and their social perception in southeastern Altiplanicie de Chiapas. Acta Zoológica Mexicana (nueva serie), 34, 1-20. https://doi.org/10.21829/azm.2018.3412111

\begin{abstract}
Current and potential distribution models were developed for four species of venomous snakes (Bothriechis aurifer, Crotalus simus, Micrurus diastema and $M$. elegans) inhabiting the southeastern area of the Altiplanicie de Chiapas. These species are included within risk categories in the NOM-059-SEMARNAT-2010, their precise distribution is unknown and they are of medical importance. The distribution models were obtained with MaxEnt algorithm, using records obtained from voucher specimens of national and foreign museums, searches in the internet, scientific literature and field sampling. In addition, occurence of each of species in the protected natural areas was determined. Interviews were also conducted to determine the perception and relationship of venomous snakes with human populations. Some geographical barriers, such as the Valle del Río Motagua, limits the distribution of $M$. elegans and B. aurifer, while the Istmo de Tehuantepec does for C. simus. Bothriechis aurifer is the most vulnerable species because of its restricted distribution and scarce occurrence in the protected natural areas. It was concluded that bites of poisonous snakes are frequent in some localities. Crotalus simus is pursued because of its traditional medicinal use. This information is important as a basis for implementing environmental education programs and management action that contribute to the conservation of the species.
\end{abstract}

Key words: Risk category; medical importance; MaxEnt; surveys; Natural Protected Areas

\title{
INTRODUCCIÓN
}

México cuenta con la mayor riqueza de serpientes venenosas del continente americano (Campbell \& Lamar, 2004), y uno de los estados más diversos es Chiapas, con 113 especies de serpientes (Johnson et al., 2015), de las cuales 20 son venenosas (Luna-Reyes et al., 2015).

En el caso particular del área sureste de la Altiplanicie de Chiapas, existen cuatro especies de serpientes venenosas con mayor riesgo de extinción, dos elápidos Micrurus diastema (Duméril, Bibron \& Duméril, 1854) y M. elegans Jan, 1858 y dos vipéridos Bothriechis aurifer (Salvin, 1860) y Crotalus simus Latreille, 1801. Además, para algunas de ellas, sus registros son escasos y se conoce poco sobre su distribución, por lo que es necesario actualizar dicho conocimiento, lo cual es fundamental para la implementación de planes de manejo y conservación (Rushton et al., 2004).

Aun cuando se han hecho algunos estudios de distribución potencial con reptiles en México, incluyendo serpientes venenosas (Ochoa-Ochoa et al., 2006), éstos no han abarcado el área de distribución completa de algunas especies, como $C$. simus. De igual manera, en los estudios que incluyen la distribución potencial de las especies y su representatividad en las áreas naturales protegidas (ANP) (Paredes-García et al., 2011), sólo se incluye a México, y no se evalúa su distribución en Centroamérica, ni representan la distribución completa de algunas especies.

Para implementar estrategias de conservación es necesario conocer la relación del hombre con la naturaleza, debido a que se trata de una variable que interviene en el deterioro ambiental (García-López et al., 2017). La manera en la que es conocido y percibido un animal por una comunidad humana, constituye parte del sistema del conocimiento tradicional, el cual puede ser considerado paralelo al sistema del conocimiento científico y genera determinada actitud de la gente hacia éste (Penguilly-Macías et al., 2009). Además, a partir del conocimiento ancestral de las comunidades indígenas se puede obtener información preliminar de la biota y aspectos generales relacionados con el estado de conservación de los 
recursos naturales en sus territorios, los cuales han sido poco explorados (Racero-Casarrubia et al., 2008). Por otra parte, las actitudes de las personas hacia las especies animales, pueden impactar de forma negativa a sus poblaciones (Enríquez-Rocha \& Rangel, 2004).

Se han realizado estudios sobre percepción social hacia las serpientes del género Crotalus en el norte de México (Gatica-Colima \& Jiménez-Castro, 2009), pero no para el resto de las especies de serpientes venenosas en el país, incluyendo el estado de Chiapas. En cuanto al uso medicinal de la fauna silvestre, se hizo un estudio en la parte central de la Altiplanicie de Chiapas (Enríquez et al., 2006) y en un ejido del sureste de esta región (Serrano-González et al., 2011); sin embargo, es poco lo que se conoce sobre los usos hacia las serpientes venenosas en el área.

El uso de modelos de distribución potencial proveen predicciones detalladas de la distribución al relacionar la presencia de las especies con predictores ambientales (Elith et al., 2006). Por todo lo anterior, el presente trabajo aborda el análisis de la distribución geográfica de cuatro especies de serpientes venenosas en riesgo de extinción de la Altiplanicie de Chiapas mediante el modelado de nicho ecológico y la percepción de las personas hacia estas especies con la aplicación de entrevistas a los pobladores. Además, se determinan las variables ambientales que influyen en su distribución y evalúa su representatividad en las ANP.

\section{MATERIALES Y MÉTODOS}

La Altiplanicie de Chiapas está constituida por una superficie accidentada y representa una de las siete regiones fisiográficas de Chiapas (Fig. 1) (Müllerried, 1957). Es un macizo montañoso de $160 \mathrm{~km}$ de largo y $75 \mathrm{~km}$ de ancho, en dirección noroeste-sureste, ubicado entre los $15^{\circ} 55^{\prime}-17^{\circ} 00^{\prime} \mathrm{N}, 91^{\circ} 30^{\prime}-93^{\circ} 10^{\prime} \mathrm{O}$ y ocupa una superficie de $6,200 \mathrm{~km}^{2}$. Los climas predominantes son templado subhúmedo y semicálido húmedo con lluvias en verano (más del $85 \%$ de la precipitación anual entre mayo y octubre) (GarcíaMartínez, 1988). La temperatura media anual oscila entre $13-15^{\circ} \mathrm{C}$ y la precipitación media anual es de 1,100-1,200 mm (García-Martínez, 1988). Los tipos de vegetación son: selva mediana y baja siempre verde, bosque deciduo, bosque de hojas planas y duras (encinar) y bosque de hojas aciculares (pinar) (Miranda, 2015).

Se realizaron visitas mensuales (febrero-agosto de 2016), en siete localidades ubicadas en el área de estudio (Fig. 1) con duración de diez días cada una. Los ejemplares fueron colectados con el permiso de la Dirección General de Vida Silvestre (SGPA/DGVS/01609/16) y depositados en la Colección Herpetológica de El Colegio de la Frontera Sur, Unidad Chetumal (ECO-CH-H).

La asignación del tipo de endemismo y categorías de riesgo de cada especie se realizó con base en la Norma Oficial Mexicana NOM-059-SEMARNAT-2010 (SEMARNAT, 2010), complementándose con los estudios de Flores-Villela (1993) y mediante consulta de la lista roja de especies amenazadas de la IUCN (IUCN, 2017), el sistema EVS (Puntaje de Vulnerabilidad Ambiental) (Johnson et al., 2015) y la Convención sobre el Comercio Internacional de Especies Amenazadas de Flora y Fauna Silvestre (CITES, 2011).

Los registros ya existentes de serpientes venenosas se obtuvieron de las bases de datos de las siguientes colecciones nacionales e internacionales: AMNH, ANSP, BYU, CAS, CM, CUMV, CNAR, ECO-CH-H, ECO-SC-H, FMNH, IHNHERP, KU, LACM, LSUMZ, MPM, MZ-UNICACH, MCZ, MZFC, OMNH, TCWC, TNHC, UF, UMMZ, USNM, UTEP, YPM y UAEH. La confiabilidad de la identidad taxonómica de los registros fue verificada por los curadores o responsables de las colecciones y personalmente se llevó a cabo la revisión en las colecciones herpetológicas ECO-SC-H, IHNHERP y MZ- 
UNICACH. Los acrónimos para los museos se encuentran en Sabaj-Pérez (2014), a excepción del Centro de Investigaciones Biológicas de la Universidad Autónoma del Estado de Hidalgo (UAEH).

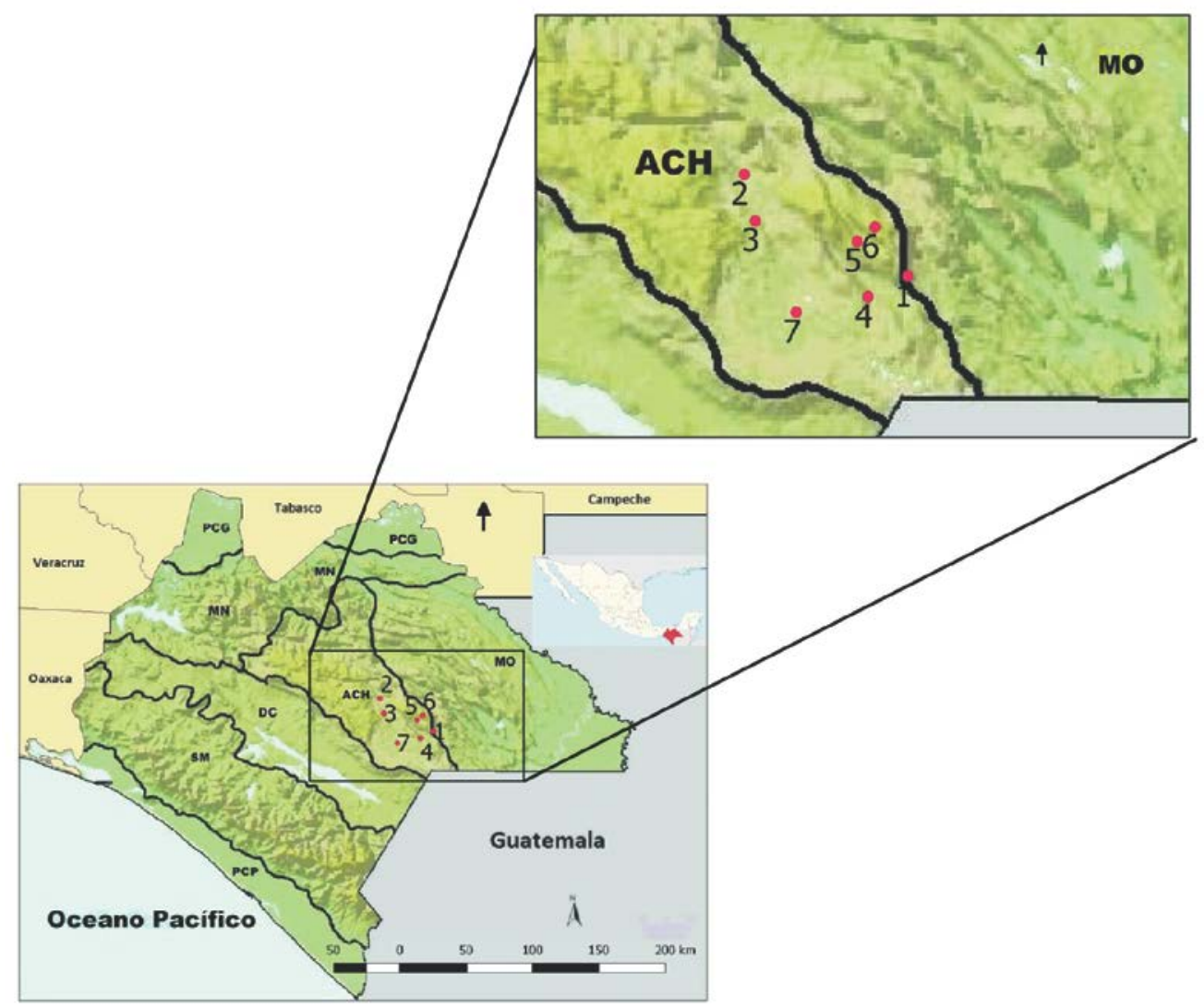

Figura 1. Área de estudio en el sureste de la Altiplanicie de Chiapas. Simbología: DC = Depresión Central, $\mathrm{ACH}=$ Altiplanicie de Chiapas, PCG = Planicie Costera del Golfo, MN = Montañas del Norte, MO = Montañas del Oriente, PCP = Planicie Costera del Pacífico, SM = Sierra Madre (tomado de Johnson et al. 2015). Localidades visitadas: 1 = Cruz del Rosario, $2=$ El Vergelito, $3=$ Bajucú, $4=$ Buenavista Pachán - El Suspiro, 5 = Santa Martha, 6 = Santiago Guelatao, 7 = Llano Grande Tintonishac.

Los registros en línea se obtuvieron de: Vernet (www.vernet.org), Global Biodiversity Information Facility (GBIF, www.gbif.org) e iNaturalist (www.inaturalist.org). Además, se usaron registros de literatura obtenidos de Campbell \& Smith (2000), Köhler (2001), Hidalgo-García (2008), Neri-Castro et al. (2013), del proyecto "Conocimiento y manejo sustentable de la herpetofauna para el fortalecimiento de la Red de Ecoturismo en el Área de Intervención de la Asociación de Silvicultores Región Miramar A. C.” y los registros obtenidos en campo durante este estudio.

Para georreferenciar los registros que sólo tenían localidad de colecta se emplearon los programas Geolocate 3.22 (Ríos \& Bart, 2010) y Google Earth Pro 7.1.5. Para depurar las bases de datos, se trazaron todos los puntos en archivos vectoriales de México y Centroamérica, en el programa Quantum GIS 2.16 (Quantum GIS Development Team, 2016) y se comparó la ubicación de los puntos de cada especie con los mapas de Campbell \& Lamar (2004), IUCN (2017) y con gráficas proyectadas en el espacio ambiental. 
Los mapas de distribución fueron generados con el programa MaxEnt (Phillips et al., 2006) (http://www.cs.princeton.edu/ schapire/maxent/), el cual es un algoritmo robusto que modela la distribución potencial de las especies por medio de la relación entre los puntos de presencia conocidos y las variables ambientales incluidas en el modelo (Phillips et al., 2006). A partir de esta relación, el algoritmo extrapola la presencia de la especie a las áreas donde no se cuenta con registros (Lindenmayer et al., 1991). Las coberturas consistieron en 19 capas de información sobre temperatura y precipitación, provenientes de WorldClim (Hijmans et al., 2005), con una resolución de $0.86 \mathrm{~km}^{2}$, las cuales fueron recortadas con el programa DIVA-GIS 7.5.0.0 (Hijmans et al., 2012), con base en los mapas de distribución de Campbell \& Lamar (2004) y de la IUCN (2017). Posteriormente, se eliminaron las capas que tuvieron alta correlación $(r>0.85)$, usando el coeficiente de Pearson. Los criterios para eliminar variables ambientales fueron dejar aquellas que pudieran interpretarse más fácilmente, tomando en cuenta el conocimiento ecológico de cada especie. El programa MaxEnt, estima además la importancia de cada variable en la distribución de las especies y el modelo generado fue validado con el área bajo la curva (AUC), derivada de la curva operada por el receptor (ROC), las cuales están implementadas en el programa (Phillips et al., 2006). Los modelos con valores entre 0.7-0.9 para los puntos de entrenamiento y prueba se consideraron razonablemente buenos y los modelos con valores $>0.9$ se catalogaron como muy buenos (Peterson et al., 2011). En el proceso de evaluación de los modelos, se usó el 70\% de los registros para entrenar el modelo y 30\% para validar, mediante validación cruzada. Para los modelos de presenciaausencia, MaxEnt calcula el valor estadístico para cada umbral, con base en una prueba de Chi-cuadrado. Una vez validado cada modelo, se emplearon todos los registros para obtener los mapas definitivos (Martínez-Meyer com. pers.), a excepción del modelo empleado para $M$. diastema en el que se usó el modelo generado con los datos de calibración (60\% de los datos, 40\% para su validación), esto para evitar un sobreajuste, por la gran cantidad de registros que se tenía para esta especie.

Para correr el modelo, se usaron las opciones por defecto de MaxEnt, como lo sugieren Phillips \& Dudik (2008), a excepción de la ventanilla de Setting/Advanced, en donde se desactivaron las casillas "Extrapolate" y "Do clamping", cuando se modelaron especies de zonas montañosas, como B. aurifer y M. elegans, para evitar extrapolar en valores extremos de las variables la distribución en lugares donde la especie no está presente (Elith et al., 2011). Se usó el formato logístico, debido a que es más fácil de interpretar, y es robusto cuando el área de distribución de una especie es desconocida (Phillips \& Dudik, 2008).

El algoritmo MaxEnt tiene incorporado un procedimiento para la selección de las variables más importantes durante la generación de los modelos de distribución potencial de las especies. Para la evaluación de las variables en el modelo y el porcentaje que aporta cada una se utilizó la prueba Jackknife (Plasencia-Vázquez et al., 2014).

Para visualizar la representatividad de las especies en las ANP, se emplearon como puntos de corte los umbrales de presencia mínima de entrenamiento, sensibilidad máxima de entrenamiento más especificidad, prueba de máxima sensibilidad más especificidad y entropía equivalente de umbrales y distribuciones originales (Liu et al., 2005). Posteriormente, a los mapas de distribución potencial les fueron eliminadas las áreas donde se conoce que el taxón no puede estar presente o no ha sido registrado, para así obtener la distribución real o área ocupada (Peterson \& Soberón, 2012), usando como criterio los mapas de Campbell \& Lamar (2004), IUCN (2017) y superponiendo capas vectoriales de las ecorregiones propuestas por Olson et al. (2001). Posteriormente, se sobrepusieron mapas digitales de las ANP federales de México y Centroamérica.

Para evaluar la percepción social sobre las especies de serpientes, se hicieron 62 entrevistas semiestructuradas a los pobladores, las cuales son una de las principales estrategias para producir datos en la investigación cualitativa (Sautu, 2003). Siete entrevistas se realizaron en el municipio de La Independencia (dos localidades) y 55 en Las Margaritas (14 localidades), dirigidas a personas entre 16 y 
93 años de edad. Se consideró entrevistar a un número similar de personas, tanto de la etnia tojolabal como de mestizos.

Inicialmente, se realizó un cuestionario piloto para saber si las preguntas eran claras y no dirigidas a una respuesta en particular (Enríquez-Rocha \& Rangel, 2004), el cual fue aplicado a habitantes de las comunidades donde existen serpientes venenosas.

Se seleccionaron informantes clave con base en su conocimiento de la fauna local, así como personas de ambos sexos con disponibilidad para participar en el estudio, y con más de cinco años de permanencia en la localidad a fin de aumentar la confiabilidad de las respuestas (Rojano et al., 2013; Álvarez et al., 2015). Se entrevistaron a personas de las localidades donde se realizó el muestreo de serpientes y aquellas de localidades aledañas, empleando un cuestionario guía, el cual estuvo conformado por 38 preguntas, distribuidas en ocho secciones: 1) Datos del encuestado, 2) Presencia e identificación de las especies, 3) Acciones al ver a una serpiente venenosa, 4) Incidencias de accidente ofídico, 5) Medidas tomadas en caso de accidente ofídico, 6) Conocimiento sobre serpientes venenosas, 7) Uso de serpientes venenosas y 8) Protección a las serpientes venenosas. Durante las entrevistas, se mostraron fotografías, tanto de serpientes inofensivas como venenosas, con el fin de que las pudieran identificar, usando la guía de Köhler (2008). Se realizó la prueba de Chi-cuadrado de Pearson para analizar la independencia entre variables cualitativas.

\section{RESULTADOS}

Se integró una base de datos con 918 registros de cuatro especies de serpientes venenosas: Micrurus diastema (603), M. elegans (76), Bothriechis aurifer (35) y Crotalus simus (204). La mayoría (904) corresponden a registros de la literatura, bases de datos en línea, informes técnicos de proyectos de investigación y ejemplares de museos, mientras que el resto (14 registros) fueron obtenidos mediante los muestreos de campo.

Las cuatro especies de serpientes venenosas reportadas en el presente trabajo se encuentran en distintas categorías de riesgo (Cuadro 1), y B. aurifer además tiene distribución restringida. Por su parte $C$. simus y $M$. diastema, tienen distribución más amplia, abarcando algunos países centroamericanos. Todos los modelos presentaron valores de AUC $>0.9$ y p $<0.05$, a excepción de $M$. diastema (AUC $=0.865$, $\mathrm{p}<$ 0.05).

Cuadro 1. Serpientes venenosas con mayor riesgo de extinción del sureste de la Altiplanicie de Chiapas y su clasificación. NOM= NOM-059-SEMARNAT-2010, $\operatorname{Pr}=$ Sujeta a Protección Especial, $\mathrm{A}=$ Amenazada; IUCN = Unión Internacional para la Conservación de la Naturaleza, LC = Preocupación Menor, VU = Vulnerable; EVS = Puntaje de Vulnerabilidad Ambiental, L = Bajo, $\mathrm{M}$ = Medio, $\mathrm{H}=$ Alto; CITES = Convención sobre el Comercio Internacional de Especies Amenazadas de Flora y Fauna Silvestres, III = Apéndice III.

\begin{tabular}{lcccc}
\hline Taxón & NOM & IUCN & EVS & CITES \\
\hline Elapidae & & & & \\
Micrurus diastema & Pr & LC & L(8) & III \\
M. elegans & Pr & LC & M(13) & -- \\
Viperidae & & & & \\
Bothriechis aurifer & A & VU & H(14) & -- \\
Crotalus simus & A & LC & M(10) & III \\
\hline
\end{tabular}


Micrurus diastema tiene una distribución extensa, principalmente en los estados de Quintana Roo y Yucatán, su límite de distribución al norte corresponde a la parte central de Veracruz y noroeste de Puebla (sur de la Huasteca y Provincia Llanuras y Lomeríos), y al sur hasta el oriente de las Montañas Chortí, en Honduras (Fig. 2 A). Se encuentra bien representada en las ANP de México, Guatemala y Belice (Fig. 2 A; Anexo 1). Para M. elegans el límite norte de su distribución es similar, desde la parte central de Veracruz y noroeste de Puebla, hacia el sureste, pasando por Oaxaca y Chiapas, hasta la parte central de Guatemala (Sierra de Santa Cruz y Chamá) (Fig. 2 C). Esta especie se encuentra mejor representada en las ANP de Chiapas (Fig. 2 C; Anexo 1). La precipitación del periodo más seco (bio14) contribuyó más a generar el mapa de distribución de M. diastema (Fig. 2 B), mientras que la temperatura media anual (bio1) lo fue para M. elegans (Fig. 2 D).

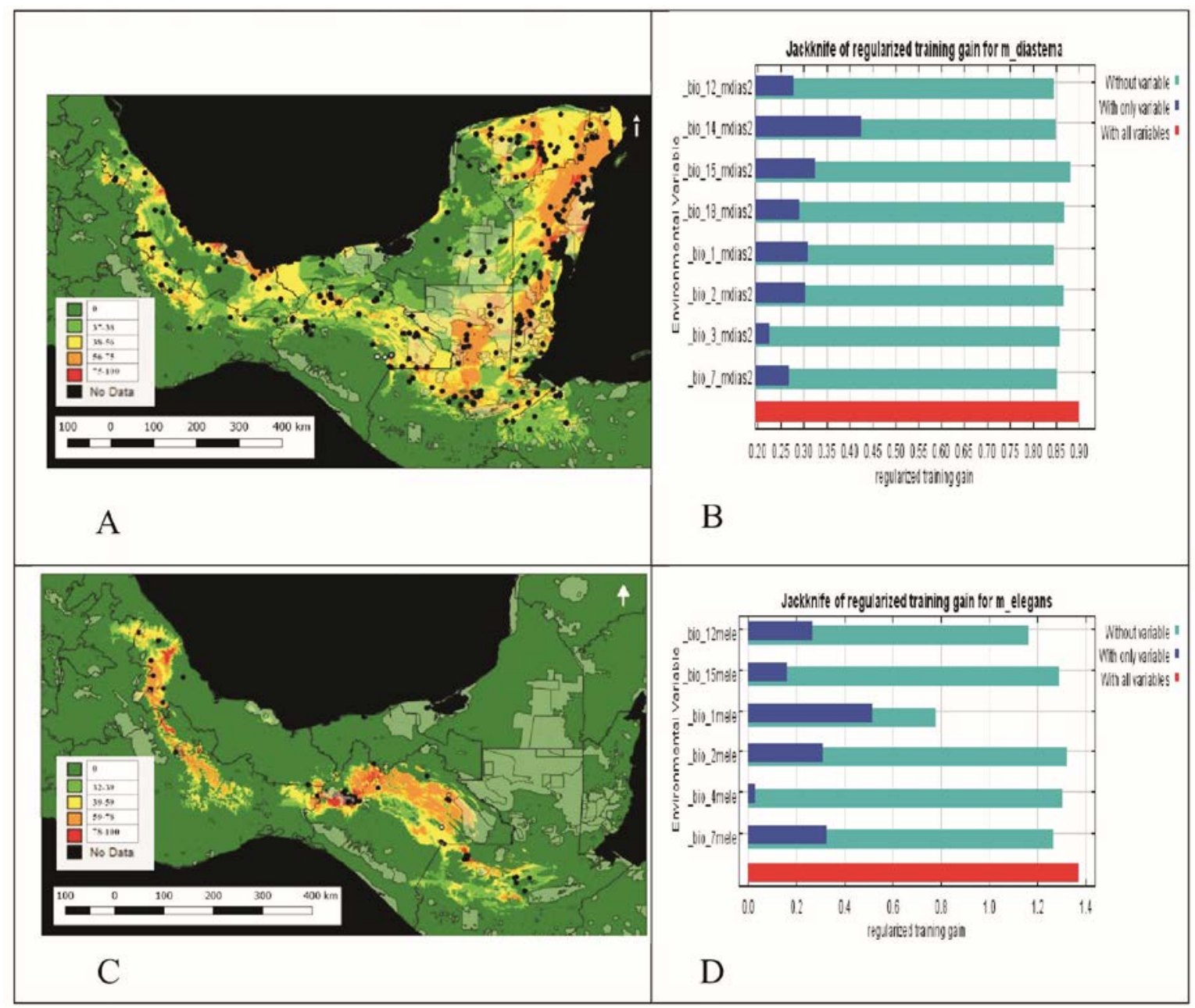

Figura 2. Área de distribución de: Micrurus diastema (A) y M. elegans (C) y su representatividad en las Áreas Naturales Protegidas de México y Centroamérica (ANP). En la escala de colores (cuadro interior), se indica la probabilidad de encontrar a la especie. Los puntos de color negro representan los registros históricos y los de color azul son las nuevas localidades de registro (registros recientes). Los polígonos de color gris son las ANP. Resultados de la prueba Jackknife para determinar la ganancia de las variables ambientales en la construcción del modelo: M. diastema (B) y M. elegans (D). 
En cuanto a los vipéridos, B. aurifer se distribuye desde el límite del área sureste de la Altiplanicie de Chiapas con las Montañas del Norte de Chiapas, hasta la Sierra de las Minas, en Guatemala (Fig. 3 A), pero con poca presencia en las ANP. Crotalus simus se distribuye desde la Llanura Costera Veracruzana y el Istmo de Tehuantepec hasta Costa Rica, en la Cordillera Volcánica Central, pero mayormente hacia la costa del Pacífico, en donde tiene buena representatividad en las ANP (Fig. 3 C, Anexo 1). Para B. aurifer, la variable temperatura media anual (bio1) contribuyó más a la generación del modelo (Fig. 3 B), y para C. simus fueron la precipitación del periodo más seco (bio14) y estacionalidad de la precipitación (bio15) (Fig. 3 D).

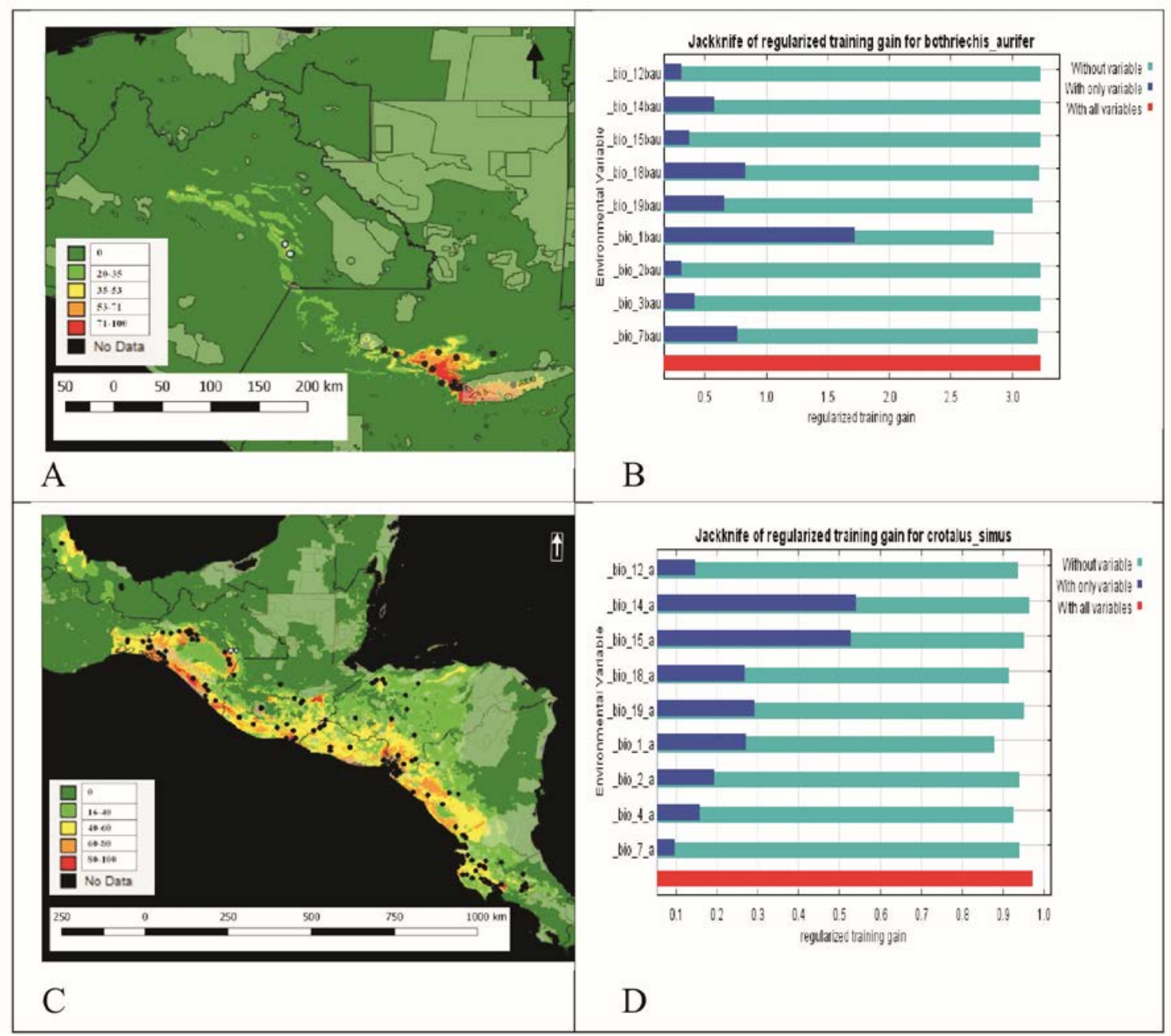

Figura 3. Área de distribución de: Bothriechis aurifer (A) y Crotalus simus (C) y su representatividad en las Áreas Naturales Protegidas de México y Centroamérica (ANP). En la escala de colores (cuadro interior), se indica la probabilidad de encontrar a la especie. Los puntos de color negro representan los registros históricos y los de color azul son las nuevas localidades de registro (registros recientes). Los polígonos de color gris son las ANP. Resultados de la prueba Jackknife para determinar la ganancia de las variables ambientales en la construcción del modelo: B. aurifer (B) y C. simus (D).

La gran mayoría de los pobladores al ver una serpiente a la que consideran venenosa la matan o intentan matarla (89\%), aunque existen diferencias significativas $\left(X^{2}=20.72, g l=4, p<0.05\right)$ en la acción que realizan en función del grado de estudios que tienen, a menor grado de estudios mayor intención por 
matarlas. No hubo diferencias significativas analizando las respuestas por grupos étnicos $\left(X^{2}=1.24, g l=\right.$ $1, p<0.27)$.

Respecto al conocimiento sobre el hábitat, $42 \%$ de los entrevistados dijeron que C. simus se encuentra en los ocotales (pinar), aunque también se mencionan milpas, zacatales, acahuales y roblares (encinar). La mayoría coincidió en que el mococh verde (B. aurifer) habita en lugares húmedos de la montaña.

El 66\% mencionó que han habido mordeduras a personas y animales domésticos causadas por víboras de cascabel. Se presentó un caso por mordedura de $C$. simus, según refieren varios pobladores, aunque el paciente al ver fotografías de las especies la identificó como Atropoides mexicanus, por lo que se debe de tener cuidado al utilizar dicho material con fines de identificación taxonómica. El mayor número de casos de mordeduras a personas correspondieron a Atropoides mexicanus (38\%) y Cerrophidion godmani (28\%), mientras que Crotalus simus (14\%), Bothriechis aurifer (10\%), Micrurus diastema (7\%) y Bothrops asper (3\%) tuvieron valores menores.

En el caso de mordeduras, el tratamiento principal consiste en ingerir bebidas alcohólicas, aunque algunos toman curarina y nauyacol (plantas). Sólo una persona mencionó que sabía o tenía noción acerca de la utilidad del suero antiviperino, actualmente conocido como faboterápico.

En diferentes localidades los pobladores matan a la víbora de cascabel (Crotalus simus), por el uso medicinal que se le atribuye, o para venderlas a personas que provienen principalmente de otras comunidades, a un precio que varía desde 50 hasta 3000 pesos dependiendo del tamaño del ejemplar. El uso medicinal más frecuente fue para curar el cáncer (53\%), aunque también se mencionaron "espanto" (16\%), dolor en general (13\%), dolor de anginas, fiebre, tos, cicatrización de heridas y para tener hijos ( $3 \%$ cada uno). Esta especie también es usada como alimento y su piel se emplea para hacer cinturones. Sólo una persona comentó que también la coralillo (Micrurus sp.) es usada con fines medicinales.

El 73\% piensan que no se deben de proteger a las serpientes venenosas, aunque hubo diferencias significativas en las respuestas entre personas con distintos niveles de escolaridad $\left(X^{2}=20.72, g l=4, p<\right.$ 0.05). Las que creen que se debe proteger cuentan con estudios (ej. primaria, secundaria, preparatoria, etc.). No hubo diferencias significativas analizando las respuestas por grupos étnicos $\left(X^{2}=0.198, g l=1, p\right.$ $<0.66)$.

\section{DISCUSIÓN}

Se obtuvieron mejores resultados en la modelación para $C$. simus, $M$. diastema y M. elegans (valores de AUC mayores a 0.9 y $p<0.05$ ) con los que se consiguieron modelos con un alto poder predictivo. Los valores para $M$. diastema (AUC $=0.865, p<0.05$ ), son considerados "razonablemente buenos" (Peterson et al. 2011); el sustento estadístico de los modelos podría estar asociado al proceso de depuración de datos, mediante el cual fueron eliminados los registros que se encontraban fuera del área conocida y espacio ambiental de las especies estudiadas.

La distribución de $M$. elegans y B. aurifer hacia el sur parece estar limitada por el Valle de Motagua, en Guatemala. Este valle ha ocasionado la divergencia de serpientes de los géneros Bothriechis, Atropoides y Cerrophidion, y actúa como una barrera geográfica para las especies de serpientes que habitan en las zonas montañosas a ambos lados del valle (Castoe et al., 2009; Daza et al., 2010).

Bothriechis aurifer es una de las especies de serpientes de la Altiplanicie de Chiapas más amenazadas, por su distribución restringida y la acelerada destrucción de su hábitat. Durante el trabajo de 
campo, se pudo apreciar que su hábitat está siendo severamente afectado, principalmente por la práctica de roza-tumba-quema. Esta tendencia de disminución de sus poblaciones por pérdida de hábitat también ha sido reportada recientemente en Guatemala (IUCN, 2017). Su distribución al norte (límites de la Altiplanicie de Chiapas con las Montañas del Norte) se encuentra limitada por la presencia de Bothriechis rowleyi, una especie emparentada (Castoe et al., 2009) y al sur por el Valle de Motagua, el cual ha actuado como una barrera geográfica para distintas especies (Castoe et al., 2009). Cabe destacar que el ejemplar observado en Santiago Guelatao y el ejemplar colectado en Felipe Carrillo Puerto (cerca de Cruz del Rosario) en el municipio de Las Margaritas, corresponden a dos nuevos registros de localidad, con los cuales su distribución se extiende aproximadamente $43 \mathrm{~km}$ al noreste (Hidalgo-García, J. A., Luna-Reyes, R., Cedeño-Vázquez, J. R. \& González-Solís, D., datos no publicados), tomado como referencia el registro previo más cercano reportado por Campbell \& Lamar (2004) en el Parque Nacional Lagunas de Montebello. La temperatura media anual fue la variable más importante para B. aurifer, que es una especie característica de lugares fríos (generalmente por encima de los $1500 \mathrm{msnm}$ ); sin embargo, la humedad también parece ser un factor importante en su distribución, ya que se encuentra en bosques mesófilos de montaña y vegetación asociada (Campbell \& Lamar, 2004).

La distribución de $C$. simus en la vertiente del Pacífico se encuentra demarcada por el Istmo de Tehuantepec, el cual ha sido una barrera geográfica para especies características de tierras bajas y altas, y separa linajes en ambos lados (Castoe et al., 2009).

Crotalus simus habita en lugares cálidos y secos y no se encuentra en las selvas húmedas (Álvarez del Toro, 1982). En este estudio, se pudo constatar que esta especie, habita en zonas más secas, a diferencia de Crotalus tzabcan, que habita en clima subhúmedo de la Península de Yucatán (Campbell \& Lamar, 2004). En el área de distribución de C. simus las lluvias llegan de manera estacional, de ahí el hecho de que la variable que contribuyó más al modelo fue la estacionalidad de la precipitación.

A pesar de que $C$. simus es una de las especies de vipéridos mejor representadas en las ANP de México (Paredes-García et al., 2011) y Centroamérica, es perseguida por las propiedades curativas que la gente le atribuye (Enríquez et al., 2006). Además, se ha reportado que se ha vuelto escasa en algunas partes de Guatemala (Campbell \& Lamar, 2004). En contraste, en Tintonishac (mpio. La Independencia) la gente adulta menciona que la población de víboras de cascabel ha aumentado en los últimos años. Esto puede deberse al cambio climático, ya que estas serpientes habitan en las regiones secas y cálidas (Álvarez del Toro, 1982) y pudieran estar encontrando condiciones más favorables en lugares que antes eran templados, debido a que son animales sensibles a los cambios de temperatura (Beaupre \& Douglas, 2009).

El cambio de uso del suelo, y en particular, la deforestación, pudieran afectar la distribución de diferentes especies de serpientes venenosas, ya que dichos factores contribuyen de manera importante a la pérdida de biodiversidad (Mas \& Flamenco-Sandoval, 2011). Por lo anterior, es necesario realizar un estudio para poder correlacionar a mayor profundidad el impacto que las poblaciones humanas han causado en la distribución y desplazamiento de las especies estudiadas, conforme han ido ocupando nuevas zonas y transformando el hábitat.

Se ha documentado que Crotalus simus es usada para atender la hinchazón del cuerpo, curar mordeduras de serpiente y gangrena (Serrano-González et al., 2011) en la Altiplanicie de Chiapas. También se han reportado usos medicinales de las víboras de cascabel para otras regiones de Chiapas (Barrasa-García, 2015), Oaxaca (Buenrostro-Silva et al., 2016), Veracruz (Gómez-Landa, 2015), Ciudad de México (Gómez-Álvarez \& Pacheco-Coronel, 2010), Puebla (Cossío-Bayugar, 2007) y Guanajuato (Leyte-Manrique et al., 2016). Esta especie se usa con fines ornamentales y comerciales en Morelos (Reyna-Rojas et al., 2015) y alimenticios en el estado de México (Monroy-Vilchis et al., 2016) y Sinaloa (Pascual-Ramos et al., 2014). Por su parte, para las especies del género Micrurus sólo ocasionalmente se 
menciona su uso con fines medicinales en la parte central de la Altiplanicie de Chiapas (Enríquez et al., 2006) y Sinaloa (Pascual-Ramos et al., 2014).

A pesar de que en México la mayoría de las mordeduras de serpientes venenosas son causadas por especies de los géneros Bothrops y Crotalus (de Roodt et al., 2005), en el área de estudio la mayoría fueron ocasionadas por Cerrophidion y Atropoides, cuya mordedura es menos severa y se cura en pocos días con remedios caseros (Álvarez del Toro, 1982; Campbell \& Lamar, 2004). Por su parte, la mordedura del mococh verde (Bothriechis aurifer) puede tardar hasta tres meses en curarse, según los entrevistados en este estudio. Al respecto, un habitante que había sufrido una mordedura de esta especie hizo referencia a que fue muy grave y, en otro caso, una persona de edad avanzada perdió una extremidad, lo cual probablemente se debió al mal tratamiento y una infección posterior. Campbell \& Lamar (2004) mencionan que $B$. aurifer ha causado mordeduras fatales en Guatemala, sin embargo, en el presente estudio, no se reportaron muertes ocasionadas por esta especie.

En cuanto a las mordeduras a animales domésticos, se tiene poca certeza del número de casos. Algunos pobladores mencionan haber visto víboras de cascabel cercanas al ganado que había muerto, sin embargo, es probable que estos animales hayan muerto de alguna otra causa, ya que es común en la región que la muerte de borregos sea provocada por parásitos, como Oestrus ovis (Perezgrovas-Garza \& CastroGámez, 2000).

Los pobladores tienen poco conocimiento sobre qué medidas tomar en caso de mordedura de serpientes venenosas. En las localidades más alejadas, las clínicas carecen de medicamentos y los pacientes tienen que viajar a los asentamientos urbanos más grandes, como la cabecera municipal. Además, la gran mayoría (94\%) no sabe para qué sirve el suero antiofídico. El nivel de desconocimiento es aún mayor que el registrado por Alemán-Mejía (2011) en Honduras (82\%) y por Fernandes-Ferreira et al. (2012) en Brasil, donde todos los entrevistados conocían el uso del suero antiofídico. Este desconocimiento, probablemente esté relacionado con la baja escolaridad de los pobladores o a la poca información ambiental que los pobladores reciben.

La mayoría de los lugareños mata serpientes por temor, aun cuando algunas personas piensan que se deben proteger. Esta percepción negativa de los pobladores hacia las serpientes ha sido reportada en comunidades nahuas de Cuetzalan del Progreso, Puebla (García-López et al., 2017); con pobladores del desierto de Chihuahua (Gatica-Colima \& Jiménez-Castro, 2009), y en asentamientos rurales de Brasil (Santos-Fita et al., 2010).

El miedo generalizado hacia las serpientes se debe en parte a que las mordeduras por algunas especies venenosas ponen en peligro la vida o causan la muerte de las personas, no obstante, éstas no representan uno de los mayores problemas de salud pública en México (de Roodt et al., 2005) o en Chiapas (Suárez-Velázquez \& Luna-Reyes, 2009). Adicionalmente, la religión judeo-cristiana (iglesia católica) ha influido en el rechazo colectivo hacia las serpientes (Sánchez-Núñez, 2006; FernandesFerreira et al., 2012), por la creencia de que son "seres condenados por Dios a la eterna penitencia” (Fernandes-Ferreira et al., 2012).

La proporción de personas en el área de estudio que creen que se deben de proteger a las serpientes venenosas es menor, comparada con lo que se reporta en el norte del país (27\% vs 46\%) (Gatica-Colima \& Jiménez-Castro, 2009). Esto puede indicar que la educación puede tener algún efecto en la percepción de las personas, y más si consideramos que Las Margaritas es uno de los municipios con mayor grado de marginación en el estado de Chiapas. Al respecto, Whitaker \& Shine (2000), Alves et al. (2014) y Pinheiro et al. (2016) mencionan que la educación es importante en el cambio de actitud de las personas hacia las serpientes. Otro aspecto que vale la pena mencionar es que al evitar que la gente mate a 
las serpientes venenosas también disminuye el riesgo de mordedura, además de evitar la pérdida de la biodiversidad (Whitaker \& Shine, 2000; Pandey et al., 2016).

Algunas creencias o mitos reflejan el desconocimiento que se tiene sobre la biología y morfología de las serpientes; por ejemplo, algunas personas que utilizan a las víboras de cascabel con fines alimenticios o medicinales seccionan a la serpiente amputándole una cuarta de longitud a partir de la punta de la cabeza y la punta de la cola antes de consumirlas, con el propósito de eliminar las partes del organismo que contienen veneno, lo cual también ha sido reportado en el norte de México (Malkin, 1962). Algunas personas entierran al animal completo porque creen también que el hueso es venenoso, lo cual es una suposición errónea, ya que las serpientes tienen las glándulas de veneno en la cabeza. Álvarez del Toro (1982), en distintas regiones de Chiapas, y Fernandes-Ferreira et al. (2012), en Brasil, reportan que la gente cree que "la coral pica por la cola". Además, existe el mito de que las corales y nauyacas persiguen a la gente, lo cual también es falso, ya que el primer recurso de sobrevivencia que utilizan las serpientes, ante la presencia de un ser humano es huir; solamente cuando se sienten acorraladas pelean para defenderse y tienden a morder a la persona que las molesta (Casas-Andreu, 2000). La existencia de mitos puede contribuir a una fuerte aversión hacia estos animales por los pobladores locales (Ceríaco, 2012; Alves et al., 2014; García-López et al., 2017).

\section{CONCLUSIONES}

Con el uso de los factores ambientales de WorldClim y el algoritmo Maxent, se pudo determinar con mayor precisión y actualizar la distribución de las serpientes venenosas que habitan en el sureste de la Altiplanicie de Chiapas, cubriendo vacíos en la distribución previamente conocida. Con este estudio, se amplió la distribución conocida de Bothriechis aurifer hacia el noreste, y también se obtuvieron nuevas localidades de registro para Micrurus diastema, M. elegans y Crotalus simus en la Altiplanicie de Chiapas. Al parecer algunas barreras geográficas (Istmo de Tehuantepec, México y Valle del Río Motagua, Guatemala) actúan limitando la distribución de M. elegans, B. aurifer y C. simus. En el área de estudio, la serpiente venenosa que se encuentra en mayor riesgo es $B$. aurifer, debido a su distribución restringida, y a que se encuentra poco representada en las ANP, además de que su hábitat sufre un grave deterioro.

La víbora de cascabel (Crotalus simus) es la especie de serpiente venenosa más perseguida, debido a las propiedades curativas que se le atribuyen, por lo que habría que hacer estudios sobre el impacto a las poblaciones de esta especie. Asimismo, el grado de escolaridad influye en la percepción de las personas hacia las serpientes venenosas. En general, los pobladores del área sureste de la Altiplanicie de Chiapas desconocen las medidas que se deben de tomar en caso de mordeduras por serpientes venenosas, por lo que es importante capacitar a las personas, así como proveer del equipo necesario para atender este tipo de accidentes en los centros de salud de la región.

Ante este panorama, es necesario incrementar los esfuerzos de conservación para las serpientes venenosas estudiadas, especialmente para Bothriechis aurifer y Crotalus simus, estableciendo estudios de monitoreo de sus poblaciones, campañas de educación ambiental y promoviendo la conservación de sus hábitats.

AgRAdECIMIENTOS. Los autores agradecen a la Dirección General de Vida Silvestre de la SEMARNAT, por el otorgamiento del permiso de colecta científica (SGPA/DGVS/01609/16) y a El Colegio de la Frontera Sur por el recurso proporcionado para los muestreos en campo. El primer autor agradece al Consejo Nacional de Ciencia y Tecnología (CONACYT) y a El Colegio de la Frontera Sur, por el recurso obtenido para la realización de la maestría. A los curadores de los siguientes museos y colecciones: AMNH, ANSP, BYU, CAS, CM, CUMV, CNAR, ECO-CH-H, ECO-SC-H, FMNH, IHNHERP, KU, 
LACM, LSUMZ, MPM, MZ-UNICACH, MCZ, MZFC, OMNH, TCWC, TNHC, UF, UAEH, UMMZ, USNM, UTEP y YPM. A todas las personas que colaboraron y permitieron realizar los muestreos en campo, especialmente a M. A. Pérez, B. Jiménez, J. C. Hidalgo, B. Vázquez, don Esdras, Rigoberto, H. Vázquez, C. Pérez, J. G. Pérez, A. Pérez, R. Guillén Moreno, R. Guillén Argueta, W. Guillén, M. A. Guillén, R. Hernández, E. Hernández, K. Hernández, A. Hernández, J. A. Vázquez, I. Celis, A. Celis, E. Torres, O. Torres, N. Morales, B. López, J. A. Vázquez, R. Hernández Morales, J. Jiménez, H. Morales, G. Morales, D. Sántis, R. Sántis, E. Gómez, B. Gómez, G. López. A C. Cundapí y R. C. Hernández por la información proporcionada. A R. Carbajal y V. H. González por sus valiosos comentarios y aclarar algunas dudas. A J. J. Schmitter Soto, J. A. Padilla Saldívar y P. J. Ramírez Barajas por la revisión de la primera versión del escrito y a dos revisores anónimos por sus valiosos comentarios y sugerencias para mejorar la versión final.

\section{LITERATURA CITADA}

Alemán-Mejía, J. B. (2011) Caracterización de reptiles y percepción local hacia las serpientes en fincas ganaderas de la subcuenca del Río Copán, Honduras. Agroforestería en las Américas, 48, 103-117.

Álvarez del Toro, M. (1982) Los reptiles de Chiapas. Gobierno del Estado de Chiapas. Tuxtla Gutiérrez. 248 pp.

Álvarez, N. S., Gerritsen, P. R. W., Gómez-Llamas, J. C. (2015) Percepciones campesinas del jaguar en diez localidades de la Reserva de la Biosfera Sierra de Manantlán en el Occidente de México: implicaciones para su conservación. Sociedad y Ambiente, 1(7), 35-54.

Alves, R. R. N., Silva, V. N., Trovão D. M. B. M., Oliveira J. V., Mourão J. S., Dias T. L. P., Alves A. G. C., Lucena, R. F. P., Barboza, R. R. D., Montenegro P. F. G. P., Vieira W. L. S., Souto W. M. S. (2014) Students' attitudes toward and knowledge about snakes in the semiarid region of Northeastern Brazil. Journal of Ethnobiology and Ethnomedicine, 10(1), 1-30. doi: 10.1186/1746-4269-10-30

Barrasa-García, S. (2015) Conocimiento y usos tradicionales de la fauna en dos comunidades campesinas de la Reserva de Biosfera de La Encrucijada, Chiapas. Etnobiología, 11(1), 16-28.

Beaupre, S. J., Douglas, L. E. (2009) Snakes as indicators and monitors of ecosystem properties. Pp. 244-61. In: Mullin S. J., Seigel R. A., (Eds.). Snakes: ecology and conservation. Nueva York and Londres: Comstock Publishing Associates, a Division of Cornell University Press.

Buenrostro-Silva, A., de la Torre, M. R., Grajales, J. G. (2016) Uso y conocimiento tradicional de la fauna silvestre por habitantes del Parque Nacional Lagunas de Chacahua, Oaxaca, México. Revista Quehacer Científico en Chiapas, 11(1), 84-94.

Campbell, J. A., Smith, E. N. (2000) A new species of arboreal pitviper from the Atlantic versant of northern Central America. Revista de Biología Tropical, 48(4), 1001-1013.

Campbell, J. A., Lamar, W. W. (2004) The venomous reptiles of the western hemisphere. Comstock Publishing, Cornell University, Ithaca, Nueva York, 475 pp.

Casas-Andreu, G. (2000) Mitos, leyendas y realidades de los reptiles en México. Ciencia Ergo Sum, 7(3), 286-291.

Castoe, T. A., Daza, J. M., Smith, E. N., Sasa, M. M., Kuch, U., Campbell, J. A., Parkinson, C. L. (2009) Comparative phylogeography of pitvipers suggests a consensus of ancient Middle American highland biogeography. Journal of Biogeography, 36(1), 88-103.

Ceríaco, L. M. P. (2012) Human attitudes towards herpetofauna: The influence of folklore and negative values on the conservation of amphibians and reptiles in Portugal. Journal of Ethnobiology and Ethnomedicine, 8(1), 8.

CITES (Convenio sobre el Comercio Internacional de Especies Amenazadas de Fauna y Flora Silvestres). (2011) Apéndices I, II and III. CITES. Ginebra, Suiza. 
Cossío-Bayugar, B. A. (2007) Conocimiento y comparación del uso de la fauna silvestre en dos comunidades ejidales del municipio de Hueytamalco, Puebla, México. Tesis de Maestría. Instituto de Ecología AC. Xalapa, Veracruz. México. 187 pp.

Daza, J. M., Castoe, T. A., Parkinson, C. L. (2010) Using regional comparative phylogeographic data from snake lineages to infer historical processes in Middle America. Ecography, 33(2), 343-354.

de Roodt, A. R., Estévez-Ramírez, J., Paniagua-Solís, J. F., Litwin, S., Carvajal-Saucedo, A., Dolab, J. A., Alagón, A. (2005) Toxicidad de venenos de serpientes de importancia médica en México. Gaceta Médica de México, 141(1), 13-21.

Elith, J., Graham, C. H., Anderson, R. P., Dudik, M., Ferrier, S., Guisan, A., Hijmans, R. J. Huettmann, F., Leathwick, J. R., Lehmann, A., Li, J., Lohmann, L. G., Loiselle, B. A., Manion, G., Moritz, C., Nakamura, M., Nakazawa, Y., Overton, J. M., Peterson, A. T., Phillips, S. J., Richardson, K., Scachetti-Pereira, R., Schapire, R. E., Soberón, J., Williams, S., Wisz, M. S., Zimmermann, N. E. (2006) Novel methods improve prediction of species' distribution from occurrence data. Ecography, 29, 129-151.

Elith, J., Phillips, S. J., Hastie, T., Dudík, M., Chee, Y. E., Yates, C. J. (2011) A statistical explanation of MaxEnt for ecologists. Diversity and Distributions, 17(1), 43-57.

Enríquez, P., Mariaca, R., Retana, O. G., Naranjo, E. J. (2006) Uso medicinal de la fauna silvestre en los Altos de Chiapas, México. Interciencia, 31(7), 491-499.

Enríquez-Rocha, P., Rangel, J. L. (2004) Conocimiento popular sobre los búhos en los alrededores de un bosque húmedo tropical protegido en Costa Rica. Etnobiología, 4, 41-53.

Fernandes-Ferreira, H., Cruz, R. L., Borges-Nojosa, D. M. \& Alves, R. R. N. (2012) Crenças associadas a serpentes no estado do Ceará, nordeste do Brasil. Sitientibus, 11(2), 153-163.

Flores-Villela, O. (1993) Riqueza de los anfibios y reptiles. Pp. 33-42. In: Flores-Villela, O. y A. Navarro S. (comps.). Biología y problemática de los vertebrados en México. Ciencias, No. Especial 7. Facultad de Ciencias, UNAM.

García-López, R., Villegas, A., Pacheco-Coronel, N., Gómez-Álvarez, G. (2017) Traditional use and perception of snakes by the Nahuas from Cuetzalan del Progreso, Puebla, Mexico. Journal of Ethnobiology and Ethnomedicine, 13(1), 6.

García-Martínez, E. (1988) Modificaciones al sistema de clasificación climática de Köppen. Instituto de Geografía, UNAM, México. 90 pp.

Gatica-Colima, A., Jiménez-Castro, A. (2009) Serpientes de cascabel: percepción por algunos pobladores del desierto chihuahuense en el estado Chihuahua. Revista Latinoamericana de Recursos Naturales, 5(3), 198-204.

Gómez-Álvarez, G., Pacheco-Coronel, N. (2010) Uso medicinal de serpientes comercializadas en dos mercados de la Ciudad de México. Etnobiología, 8(1), 51-58.

Gómez-Landa, J. N. (2015) Uso de fauna silvestre en el ejido Pinoltepec, Municipio de Emiliano Zapata, Ver. Tesis de Licenciatura. Universidad Veracruzana. Xalapa, Veracruz. 42 pp.

Hernández, P. A., Graham, C. H., Master, L. L., Albert, D. L. (2006) The effect of sample size and species characteristics on performance of different species distribution modeling methods. Ecography, 29(5), 773-785.

Hidalgo-García, J. A. (2008) Diversidad de reptiles del Parque Nacional Cañón del Sumidero, Chiapas, México. Tesis de Licenciatura. Escuela de Biología. Universidad de Ciencias y Artes de Chiapas. Tuxtla Gutiérrez, Chiapas. 194 pp.

Hijmans, R. J., Cameron, S. E., Parra, J. L., Jones, P. G., Jarvis, A. (2005) Very high resolution interpolated climate surfaces for global land areas. International Journal of Climatology, 25, 1965-1978.

Hijmans, R. J., Guarino, L., Mathur, P. (2012) DIVA-GIS ver. 7.5. Manual. On line: http:www.divagis.org/docs/DIVA-GIS_manual_7.pdf.

IUCN. (2017) The IUCN Red List of Threatened Species. Version 2017-3. <http://www.iucnredlist.org>. (Consultado el 9 Septiembre 2017). 
Johnson, J. D., Mata-Silva, V., García Padilla, E., Wilson, L. D. (2015) The herpetofauna of Chiapas, Mexico: composition, distribution and conservation. Mesoamerican Herpetology, 2, 272-329.

Köhler, G. (2001) Anfibios y reptiles de Nicaragua. Herpeton. Offenbach, Alemania, 208 pp.

Köhler, G. (2008) Reptiles of Central America. Second edition. HerpetonVerlag, Offenbach, Alemania, $400 \mathrm{pp}$.

Leyte-Manrique, A., Álvarez, N. G., Hernández-Navarro, E. M. (2016) Percepción cultural de la herpetofauna en tres comunidades rurales del municipio de Irapuato, Guanajuato, México. Etnobiología, 14(1), 73-84.

Lindenmayer, D., Nix, H., McMahon, J., Hutchinson, M., Tanton, M. (1991). The conservation of leadbeather's possum, Gymnobelideus leadbeateri McCoy, a case study of the use of bioclimatic modelling. Journal of Biogeography, 18, 371-383.

Liu, C., Berry, P. M., Dawson, T. P., Pearson, R. G. (2005) Selecting thresholds of occurrence in the prediction of species distributions. Ecography, 28(3), 385-393.

Luna-Reyes, R., Canseco-Márquez, L., Hernández-García, E. (2013) Los reptiles. Pp. 319-328. In: Comisión Nacional para el Conocimiento y Uso de la Biodiversidad (Ed.). La biodiversidad de Chiapas, Estudio de Estado: México. Comisión Nacional para el Conocimiento y Uso de la Biodiversidad (CONABIO)/Gobierno del Estado de Chiapas.

Malkin, B. (1962) Seri ethnozoology. Ocassional Papers of the Idaho State Collection and Museum, 7, 58.

Mas, J. F., Flamenco-Sandoval, A. (2011) Modelación de los cambios de coberturas/uso del suelo en una región tropical de México. GeoTropico, 5(1), 1-24.

Miranda, F. (2015) La vegetación de Chiapas. Tomo 1. Cuarta Edición. Universidad de Ciencias y Artes de Chiapas. Tuxtla Gutiérrez, Chiapas. 305 pp.

Monroy-Vilchis, O., Cabrera, L., Suárez, P., Zarco-González, M. M., Rodríguez-Soto, C., Urios, V. (2016) Uso tradicional de vertebrados silvestres en la Sierra Nanchititla, México. Interciencia, 33(4), 308-313.

Müllerried, F. K. G. (1957) Geología de Chiapas. Ediciones del Gobierno del Estado. Tuxtla Gutiérrez, Chiapas. 180 pp.

Neri-Castro, E., Lomonte, B., Gutiérrez, M. del C., Alagón, A., Gutiérrez, J. M. (2013) Intraspecies variation in the venom of the rattlesnake Crotalus simus from Mexico: Different expression of crotoxin results in highly variable toxicity in the venoms of three subspecies. Journal of Proteomics, 87, 103-121.

Ochoa-Ochoa, L., Flores-Villela, O., García-Vázquez U., Correa-Cano, M., Canseco-Márquez, L. (2006) Áreas potenciales de la distribución de la herpetofauna en México. Especies: Bothriechis aurifer, Crotalus simus, M. diastema, M. elegans. Proyecto DS009: Áreas potenciales de distribución y GAP análisis de la herpetofauna en México. CONABIO, México. $<$ http://www.conabio.gob.mx/informacion/gis/layouts/trace_ornagw.gif $>$ (consultado el 7 de diciembre de 2015).

Olson, D. M., Dinerstein, E., Wikramanayake, E. D., Burgess, N. D., Powell, G. V., Underwood, E. C., Loucks, C. J. (2001) Terrestrial ecoregions of the World: A new map of life on Earth a new global map of terrestrial ecoregions provides an innovative tool for conserving biodiversity. BioScience, 51(11), 933-938.

Pandey, D. P., Pandey, G. S., Devkota, K., Goode, M. (2016) Public perceptions of snakes and snakebite management: implications for conservation and human health in southern Nepal. Journal of Ethnobiology and Ethnomedicine, 12(1), 22. Doi: 10.1186/s13002-016-0092-0

Paredes-García, D. M., Ramírez-Bautista, A., Martínez-Morales, M. A. (2011) Distribution and representation of Crotalus species within the natural protected areas of Mexico. Revista Mexicana de Biodiversidad, 82, 689-700.

Pascual-Ramos, E., Medina-Torres, S. M., Sandoval-Forero, E. A., Lara-Ponce, E., Piña-Ruíz, H. H., Martínez-Ruíz, R., Rojo-Martínez, G. E. (2014) Uso de reptiles entre Yoremes y Yoris en el municipio de El Fuerte, Sinaloa. Ra Ximhai, 10(3), 195-208. 
Penguilly-Macías, M., Moreno-Fuentes, A., Goyenechea, I., Espinoza-Pineda, G. (2009) Percepción acerca de las lagartijas consideradas nocivas por algunos otomíes, nahuas, tepehuas y mestizos en el estado de Hidalgo, México. Pp. 99-105. In: Moreno, A., Valadéz, R., Pulido, M. T., Mariaca, R., Mejía, P. \& Gutiérrez Santillán, T. V. (Eds.). Etnobiología y sistemas biocognitivos tradicionales: paradigmas en la conservación biológica y el fortalecimiento cultural. Universidad Autónoma del Estado de Hidalgo, México, Asociación Etnobiológica Mexicana y Sociedad Latinoamericana de Etnobiología, México.

Perezgrovas-Garza, R., Castro-Gámez, H. (2000) El borrego Chiapas y el sistema tradicional de manejo de ovinos entre las pastora tzotziles. Archivos de Zootecnia, 49(187), 391-403.

Peterson, A. T., Soberón, J., Pearson, R. G., Anderson, R. P., Martínez Meyer, E., Nakamura, M., Araújo, M. B. (2011) Ecological Niches and Geographic Distributions. Princeton University Press. Nueva Jersey, $314 \mathrm{pp}$.

Peterson, A. T., Soberón, J. (2012) Species distribution modeling and ecological niche modeling: getting the concepts right. Natureza \& Conservação, 10(2), 102-107.

Phillips, S. J., Anderson, R. P., Schapire, R. E. (2006) Maximum entropy modeling of species geographic distributions. Ecological Modelling, 190, 231-259.

Phillips, S. J., Dudík, M. (2008) Modeling of species distributions with MaxEnt: new extensions and a comprehensive evaluation. Ecography, 31(2), 161-175.

Pinheiro, L. T, Mota-Rodríguez, J. F., Borges-Nojosa, D. M. (2016) Formal education, previous interaction and perception influence the attitudes of people toward the conservation of snakes in a large urban center northeastern Brazil. Journal of Ethnobiology and Ethnomedicine, 12(1), 25. Doi: 10.1186/s13002-016-0096-9

Plasencia-Vázquez, A. H., Escalona-Segura, G., Esparza-Olguín, L. G. (2014) Modelación de la distribución geográfica potencial de dos especies de psitácidos neotropicales utilizando variables climáticas y topográficas. Acta Zoológica Mexicana (nueva serie), 30(3), 471-490.

Quantum GIS, Development Team, (2016) Quantum GIS (Geographic Information System). Open Source Geospatial Foundation Project. <http://www.qgis.org> (Consultado el 18 de agosto de 2016).

Racero-Casarrubia, J., Vidal, C. C., Ruiz, O. D., Bastelleros, J. (2008) Percepción y patrones de uso de la fauna silvestre o comunidades indígenas Embera-Katíos en la cuenca del río San Jorge, zona amortiguadora del PNN-Paramillo. Revista de Estudios Sociales, 31, 118-131.

Reyna-Rojas, M. A., Flores, A. G., Neri-Castro, E. E., Cano, A. A., Martínez, R. M. (2015) Conocimiento etnoherpetológico de dos comunidades aledañas a la reserva estatal Sierra de Montenegro, Morelos, México. Etnobiología, 13(2), 37-48.

Ríos, N. E., Bart, H. L. (2010) GEOLocate (Version 3.22) [computer software]. Belle Chasse, L.A: Tulane University Museum of Natural History.

Rojano, C., Padilla, H., Almentero, E., Álvarez, G. (2013) Percepciones y usos de los Xenarthra e implicaciones para su conservación en Pedraza, Magdalena, Colombia. Edentata, 14, 58-65.

Rushton, S. P., Ormerod, S. J., Kerby, G. (2004) New paradigms for modelling species distributions? Journal of Applied Ecology, 41, 193-200.

Sabaj-Pérez, M. H. (2014) Standard symbolic codes for institutional resource collections in herpetology and ichthyology: an online reference. Version 5.0. American Society of Ichthyologists and Herpetologists, Washington, D.C. <http://www.asih.org> (Consultado el 04 Noviembre, 2016).

Sánchez-Núñez, E. (2006) Conocimiento tradicional mazahua de la herpetofauna: un estudio etnozoológico en la Reserva de la Biósfera Mariposa Monarca, México. Estudios Sociales: Revista de Investigación Científica, 14(28), 43-66.

Santos-Fita, D., Costa-Neto, E. M., Sciavetti, A. (2010) 'Offensive' snakes: cultural beliefs and practices related to snakebites in a Brazilian rural settlement. Journal of Ethnobiology and Ethnomedicine, 6(1), 1-13. Doi: 10.1186/1746-4269-6-13

Sautu, R. (2003) Todo es teoría. Lumière Ediciones.Buenos Aires. 98 pp. 
SEMARNAT (Secretaría de Medio Ambiente y Recursos Naturales) (2010) Norma Oficial Mexicana NOM-059-SEMARNAT-2010. Protección ambiental-Especies nativas de México de flora y fauna silvestre-Categorías de riesgo y especificaciones para su inclusión, exclusión o cambio-Lista de especies en riesgo. Diario Oficial de la Federación, 30 de diciembre 2010, Segunda Sección, México.

Serrano-González, R., Guerrero, F., Serrano, R. (2011) Animales medicinales y agoreros entre Tzotziles y Tojolabales. Estudios Mesoamericanos, 11, 29-42.

Suárez-Velázquez, A., Luna-Reyes, R. (2009) Mordeduras por serpientes en Chiapas. Instituto de Salud Pública en el estado de Chiapas/ Régimen Estatal de Protección Social en Salud en Chiapas. Gobierno de Chiapas. Tuxtla Gutiérrez, Chiapas, México. 120 pp.

Whitaker, P. B., Shine, R. (2000) Sources of mortality of large elapid snakes in an agricultural landscape. Journal of Herpetology, 34(1), 121-128. 
Anexo 1. Principales Áreas Naturales Protegidas (ANP) donde se distribuyen las serpientes venenosas con mayor riesgo de extinción del sureste de la Altiplanicie de Chiapas. Abreviaturas: Micrurus diastema (M.d.), Micrurus elegans (M.e.), Bothriechis aurifer (B.a.) y Crotalus simus (C.s.).

\begin{tabular}{|c|c|c|c|c|}
\hline Área Natural Protegida & $\begin{array}{c}\text { Estado o } \\
\text { Departamento }\end{array}$ & Designación & $\begin{array}{c}\text { Área } \\
\text { (ha) }\end{array}$ & Especies \\
\hline \multicolumn{5}{|l|}{ MÉXICO } \\
\hline Anillo de Cenotes & Yucatán & Reserva Estatal & 214,678 & M.d. \\
\hline $\begin{array}{l}\text { Reserva Estatal Biocultural } \\
\text { del Puuc }\end{array}$ & Yucatán & Reserva Estatal & 135,893 & M.d. \\
\hline Yulum Balam & Quintana Roo & $\begin{array}{c}\text { Área de Protección de Flora y } \\
\text { Fauna }\end{array}$ & 154,052 & M.d. \\
\hline Sian Ka'an & Quintana Roo & Reserva de la Biósfera & 528,147 & M.d. \\
\hline Santuario del Manatí & Quintana Roo & $\begin{array}{l}\text { Zona Sujeta a Conservación } \\
\text { Ecológica }\end{array}$ & 277,316 & M.d. \\
\hline Balam Ka’ax & Quintana Roo & $\begin{array}{c}\text { Área de Protección de Flora y } \\
\text { Fauna }\end{array}$ & 128,390 & M.d. \\
\hline Calakmul & Campeche & Reserva de la Biósfera & 723,185 & M.d. \\
\hline Laguna de Términos & Campeche & $\begin{array}{c}\text { Área de Protección de Flora y } \\
\text { Fauna }\end{array}$ & 705,105 & M.d. \\
\hline Sierra de Tabasco & Tabasco & Reserva Ecológica & 15,113 & M.d., M.e. \\
\hline Pantanos de Centla & Tabasco & Reserva de la Biósfera & 302,706 & M.d. \\
\hline Cañón del Usumacinta & Tabasco & $\begin{array}{c}\text { Área de Protección de Flora y } \\
\text { Fauna }\end{array}$ & 46,128 & M.d. \\
\hline Reserva Ecológica Filobobos & Veracruz & Área Natural Protegida & 10,528 & M.d., M.e. \\
\hline Los Tuxtlas & Veracruz & Reserva de la Biósfera & 155,122 & M.d., M.e. \\
\hline $\begin{array}{l}\text { Parque Nacional Cañón del } \\
\text { Río Blanco }\end{array}$ & Veracruz & Parque Nacional & 48,799 & M.d., M.e. \\
\hline Santiago Tlatepusco & Oaxaca & $\begin{array}{l}\text { Área Destinada Voluntariamente a } \\
\text { la Conservación }\end{array}$ & 4,300 & M.d., M.e. \\
\hline San Pedro Tlatepusco & Oaxaca & $\begin{array}{c}\text { Área Destinada Voluntariamente a } \\
\text { la Conservación }\end{array}$ & 5,050 & M.d., M.e. \\
\hline El Cordón del Retán & Oaxaca & $\begin{array}{c}\text { Área Destinada Voluntariamente a } \\
\text { la Conservación }\end{array}$ & 15,328 & M.d., C.s. \\
\hline La Sepultura & Chiapas & Reserva de la Biósfera & 167,309 & C.s. \\
\hline El Triunfo & Chiapas & Reserva de la Biósfera & 119,177 & C.s. \\
\hline La Concordia-Zaragoza & Chiapas & Áreas Naturales Típicas & 16,409 & C.s. \\
\hline El Ocote & Chiapas & Reserva de la Biósfera & 121,000 & C.s, M.d., M.e. \\
\hline La Pera & Chiapas & $\begin{array}{c}\text { Zona Sujeta a Conservación } \\
\text { Ecológica }\end{array}$ & 7,506 & C.s., M.d., M.e. \\
\hline Cerro Мeyapac & Chiapas & $\begin{array}{c}\text { Zona Sujeta a Conservación } \\
\text { Ecológica }\end{array}$ & 1,742 & C.s., M.e. \\
\hline Cañón del Sumidero & Chiapas & Parque Nacional & 21,789 & C.s., M.e. \\
\hline La Encrucijada & Chiapas & Reserva de la Biósfera & 144,868 & C.s. \\
\hline Santa Ana & Chiapas & $\begin{array}{l}\text { Zona Sujeta a Conservación } \\
\text { Ecológica }\end{array}$ & 512 & M.d. \\
\hline Chanal & Chiapas & Áreas Naturales Típicas & 4,242 & M.e. \\
\hline Sistema Lagunar Catazajá & Chiapas & $\begin{array}{c}\text { Zona Sujeta a Conservación } \\
\text { Ecológica }\end{array}$ & 41,058 & M.d. \\
\hline Palenque & Chiapas & Parque Nacional & 1,791 & M.d. \\
\hline $\begin{array}{l}\text { Cascadas de Agua } \\
\text { Azul }\end{array}$ & Chiapas & $\begin{array}{l}\text { Área de Protección de } \\
\text { Flora y Fauna }\end{array}$ & 2,612 & M.d. \\
\hline Nahá-Metzabok & Chiapas & $\begin{array}{l}\text { Área de Protección de Flora y } \\
\text { Fauna }\end{array}$ & 3,847 & M.d., M.e. \\
\hline
\end{tabular}




\begin{tabular}{|c|c|c|c|c|}
\hline Área Natural Protegida & $\begin{array}{c}\text { Estado o } \\
\text { Departamento }\end{array}$ & Designación & $\begin{array}{l}\text { Área } \\
\text { (ha) }\end{array}$ & Especies \\
\hline Lagunas de Montebello & Chiapas & Parque Nacional & 6,022 & $\begin{array}{l}\text { B.a., C.s., M.d., } \\
\text { M.e., }\end{array}$ \\
\hline Montes Azules & Chiapas & Reserva de la Biósfera & 331,200 & M.d., M.e. \\
\hline Volcán Tacaná & Chiapas & Reserva de la Biósfera & 6,378 & C.s. \\
\hline \multicolumn{5}{|l|}{ BELICE } \\
\hline Río Bravo & Orange Walk & Private Reserve & 104,896 & M.d. \\
\hline Bladen & Toledo & Nature Reserve & 40,336 & M.d. \\
\hline Deep River & Toledo & Forest Reserve & 31,797 & M.d. \\
\hline Columbia River & Toledo & Forest Reserve & 60,037 & M.d. \\
\hline Paynes Creek & Toledo & National Park & 15,248 & M.d. \\
\hline Manatee & Belize & Forest Reserve & 36,393 & M.d. \\
\hline Fresh Water Creek & Belize & Forest Reserve & 11,359 & M.d. \\
\hline Sibun & Cayo & Forest Reserve & 42,974 & M.d. \\
\hline Chiquibul & Cayo & National Park & 107,300 & M.d. \\
\hline Mountain Pine Ridge & Cayo & $\begin{array}{c}\text { Mountain Pine Ridge Forest } \\
\text { Reserve }\end{array}$ & 43,081 & M.d. \\
\hline Cockscomb Basin & Stann Creek & $\begin{array}{l}\text { Cockscomb Basin Wildlife } \\
\text { Sanctuary }\end{array}$ & 35,336 & M.d. \\
\hline Sitee River & Stann Creek & Forest Reserve & 38,008 & M.d. \\
\hline \multicolumn{5}{|l|}{ GUATEMALA } \\
\hline Cerro San Gil & Izabal & Reserva Protectora de Manantiales & 47,432 & M.d. \\
\hline Río Sarstun & Izabal & Zona de Uso Múltiple & 35,200 & M.d. \\
\hline Punta de Namabique & Izabal & $\begin{array}{l}\text { Ramsar Site, Wetland of } \\
\text { International Importance }\end{array}$ & 151,878 & M.d. \\
\hline Bocas del Polochic & Izabal & Refugio de Vida Silvestre & 20,760 & M.d. \\
\hline Manchón Guamuchal & $\begin{array}{l}\text { Retalhuleu, San } \\
\text { Marcos }\end{array}$ & $\begin{array}{l}\text { Ramsar Site, Wetland of } \\
\text { International Importance }\end{array}$ & 13,500 & C.s. \\
\hline Volcán Tajumulco & San Marcos & Zona de Veda Definitiva & 13,031 & C.s. \\
\hline Cuenca del Lago Atitlán & Sololá & Zona de Uso Múltiple & 122,900 & C.s. \\
\hline Volcán Fuego & Sacatepéquez & Zona de Veda Definitiva & 12,150 & C.s. \\
\hline Volcán Agua & $\begin{array}{l}\text { Sacatepéquez. } \\
\text { Escuintla, } \\
\text { Chimaltenango }\end{array}$ & Zona de Veda Definitiva & 12,600 & C.s. \\
\hline $\begin{array}{l}\text { Reserva Natural de } \\
\text { Monterrico }\end{array}$ & Santa Rosa & Zona de Uso Múltiple & 2,800 & C.s. \\
\hline Volcán Tecuamburro & Santa Rosa & Zona de Veda Definitiva & 5,120 & C.s. \\
\hline Trifinio & Jutiapa & Reserva de la Biósfera & 22,178 & C.s., M.d. \\
\hline Maya & El Petén & Reserva de la Biósfera & $2,160,200$ & M.d. \\
\hline Ixil Visis Cabá & El Quiché & Reserva de la Biósfera & 45,000 & M.d., M.e. \\
\hline Finca Rústica Chimel & El Quiché & Reserva Natural Privada & 2,000 & B.a., M.d., M.e. \\
\hline Laguna Lachúa & Alta Verapaz & Parque Nacional & 14,500 & M.d., M.e. \\
\hline Cerro Verde & Baja Verapaz & Reserva Natural Privada & 585 & B.a. \\
\hline Santa Rosa y Llano Largo & Baja Verapaz & Reserva Natural Privada & 1,083 & B.a., M.d, M.e., \\
\hline Mario Dary & Baja Verapaz & Biotopo Protegido & 1,022 & B.a. \\
\hline Sierra de las Minas & Baja Verapaz & Reserva de la Biósfera & 242,642 & B.a., C.s., M.d. \\
\hline Sipacate-Naranjo & Escuintla & Parque Nacional & 2,000 & C.s. \\
\hline \multicolumn{5}{|l|}{ EL SALVADOR } \\
\hline & Santa Ana & Parque Nacional & 1,973 & C.s. \\
\hline Apaneca-Llamatepec & Ahuachapán & Reserva de la Biósfera & 59,056 & C.s. \\
\hline
\end{tabular}




\begin{tabular}{|c|c|c|c|c|}
\hline Área Natural Protegida & $\begin{array}{c}\text { Estado o } \\
\text { Departamento }\end{array}$ & Designación & $\begin{array}{l}\text { Área } \\
\text { (ha) }\end{array}$ & Especies \\
\hline Embalse Cerrón Grande & $\begin{array}{c}\text { Chalatenango, San } \\
\text { Salvador, Cuscatlán, } \\
\text { Cabañas }\end{array}$ & $\begin{array}{l}\text { Ramsar Site, Wetland of } \\
\text { International Importance }\end{array}$ & 60,698 & C.s. \\
\hline Bahía de Jiquilisco & Usulután & $\begin{array}{l}\text { Ramsar Site, Wetland of } \\
\text { International Importance }\end{array}$ & 63,500 & C.s. \\
\hline Laguna de Olomega & $\begin{array}{l}\text { San Miguel, La } \\
\text { Unión }\end{array}$ & $\begin{array}{l}\text { Ramsar Site, Wetland of } \\
\text { International Importance }\end{array}$ & 7,556 & C.s. \\
\hline \multicolumn{5}{|l|}{ HONDURAS } \\
\hline $\begin{array}{l}\text { Montecristo Trifinio } \\
\text { El Pital }\end{array}$ & $\begin{array}{l}\text { Ocotepeque } \\
\text { Ocotepeque }\end{array}$ & $\begin{array}{c}\text { Parque Nacional } \\
\text { Reserva Biológica }\end{array}$ & $\begin{array}{c}68,215 \\
2,677\end{array}$ & $\begin{array}{l}\text { C.s. } \\
\text { C.s. }\end{array}$ \\
\hline Celaque & Ocotepeque & Parque Nacional & 26,266 & C.s., M.d. \\
\hline El Guisayote & Ocotepeque & Reserva Biológica & 14,081 & C.s. \\
\hline Erapuca & Ocotepeque, Copán & Refugio de Vida Silvestre & 6,522 & C.s., M.d. \\
\hline Opalaca & Intibucá, Lempira & Reserva Biológica & 25,892 & C.s., M.d. \\
\hline El Jilguero & La Paz & Área Productora de Agua & 43,946 & C.s. \\
\hline Sabanetas & La Paz & Reserva Biológica & 8,198 & C.s. \\
\hline Montaña de Comayagua & Comayagua & Parque Nacional & 29,767 & C.s. \\
\hline Montecillos & Comayagua & Reserva Biológica & 20,333 & C.s. \\
\hline Cerro Azul Meámbar & Comayagua & Parque Nacional & 31,339 & C.s., M.d. \\
\hline Santa Bárbara & Santa Bárbara & Parque Nacional & 13,951 & C.s. \\
\hline Lago de Yojoa & Cortés & Área de Uso Múltiple & 30,141 & C.s., M.d. \\
\hline Sierra de Río Tinto & Cortés & Parque Nacional & 88,089 & C.s. \\
\hline Cusuco & Cortés & Parque Nacional & 17,704 & C.s., M.d. \\
\hline Cerro Azul & Copán & Parque Nacional & 12,083 & M.d. \\
\hline La Muralla & Olancho & Parque Nacional & 26,904 & C.s. \\
\hline Montaña de Botaderos & $\begin{array}{l}\text { Olancho, Colón, } \\
\text { Yoro }\end{array}$ & Parque Nacional & 96,755 & C.s. \\
\hline Pico Pijol & Atlántida & Parque Nacional & 11,508 & C.s. \\
\hline Pico Bonito & Atlántida & Parque Nacional & 107,107 & C.s. \\
\hline Texiguat & Atlántida, Yoro & Refugio de Vida Silvestre & 33,267 & C.s. \\
\hline La Tigra & Francisco Morazán & Parque Nacional & 24,341 & C.s. \\
\hline Sierra de Agalta & Francisco Morazán & Parque Nacional & 73,724 & C.s. \\
\hline Yerbabuena & Francisco Morazán & Reserva Biológica & 3,522 & C.s. \\
\hline Río Plátano & Gracias a Dios & Parque Nacional & 832,335 & C.s. \\
\hline \multicolumn{5}{|l|}{ NICARAGUA } \\
\hline Volcán Cosiguina & Chinadega & Reserva Natural & 13,160 & C.s. \\
\hline Estero Real & Chinadega, León & Reserva Natural & 55,000 & C.s. \\
\hline Cordillera Dipilto y Jalapa & Nueva Segobia & Reserva Natural & 41,200 & C.s. \\
\hline Bosawás & Jinotega & Reserva Natural & 685,614 & C.s. \\
\hline Miraflor & Estelí & Paisaje terrestre & 17,413 & C.s. \\
\hline Sureste de Nicaragua & Río San Juan & Reserva de la Biósfera & 767,710 & C.s. \\
\hline \multicolumn{5}{|l|}{ COSTA RICA } \\
\hline Guanacaste & Guanacaste & Parque Nacional & 33,780 & C.s. \\
\hline Miravalles & Guanacaste & Zona Protectora & 11,670 & C.s. \\
\hline Diria & Guanacaste & Parque Nacional & 5,426 & C.s. \\
\hline Palo Verde & Guanacaste & Parque Nacional & 18,291 & C.s. \\
\hline Península de Nicoya & Guanacaste & Zona Protectora & 21,317 & C.s. \\
\hline Tenorio & $\begin{array}{l}\text { Alajuela, } \\
\text { Guanacaste }\end{array}$ & Zona Protectora & 5,588 & C.s. \\
\hline Arenal Monteverde & Puntarenas & Zona Protectora & 28,314 & C.s. \\
\hline Cordillera Volcánica Central & Valle Central & Reserva Forestal & 61,278 & C.s. \\
\hline
\end{tabular}

